\title{
A magnetic bottle time-of-flight electron spectrometer suitable for continuous ionization sources
}

\author{
Cite as: Rev. Sci. Instrum. 90, 063105 (2019); doi: 10.1063/1.5097388 \\ Submitted: 25 March 2019 • Accepted: 23 May 2019 • \\ Published Online: 13 June 2019
}

Christoph Strobel, ${ }^{1}$ Gerd Gantefoer, ${ }^{1, a)}$ (D) Andras Bodi, ${ }^{2}$ (D) and Patrick Hemberger ${ }^{2}$ (D)

\author{
AFFILIATIONS \\ ${ }^{1}$ Department of Physics, University of Konstanz, 78457 Konstanz, Germany \\ ${ }^{2}$ Laboratory for Synchrotron Radiation and Femtochemistry, Paul Scherrer Institute, 5232 Villigen, Switzerland
}

${ }^{\text {a) }}$ Author to whom correspondence should be addressed: gerd.gantefoer@uni-konstanz.de

\begin{abstract}
We present a newly developed magnetic-bottle time-of-flight electron spectrometer suitable for continuous or quasicontinuous photoionization sources such as synchrotrons. A strong magnetic field collects almost all photoelectrons from a well-defined ionization volume and quantitatively suppresses background electrons which originate outside of this interaction region. Although it is a pulsed instrument, a relatively high duty cycle is achieved by storing the photoelectrons generated between two cycles in an electromagnetic trap. This makes the new instrument suitable for experiments with very low sample densities. Another advantage is the high energy resolution, $50 \mathrm{meV}$ in the first version of the spectrometer described here, which simply depends on the length of the time-of-flight instrument.
\end{abstract}

Published under license by AIP Publishing. https://doi.org/10.1063/1.5097388

\section{INTRODUCTION}

Photoelectron spectroscopy is one of the most fundamental experimental techniques to study the physical and chemical properties of atoms, molecules, clusters, nanoparticles, surfaces, and bulk materials. ${ }^{1-5}$ In the most common version, the electrons are created by irradiation of the target with light with a well-defined photon energy and their kinetic energy is measured. Disregarding angular distributions, four parameters are crucial for a successful photoelectron spectroscopy experiment:

- energy resolution,

- electron collection efficiency,

- duty cycle, and

- signal to noise ratio.

The duty cycle gives the fraction of the measurement time when the spectrometer is live. Pulsed sample and ionization sources are best matched with low duty cycle spectrometers, while continuous sample and ionization sources require a high duty cycle experiment. The collection efficiency gives the fraction of the electrons that reach the detector during this time. All four parameters depend on the type of electron spectrometer. The most common one is the hemispherical analyzer. It can achieve an excellent energy resolution of a few millielectronvolts, but the overall detection efficiency is limited $(<1 \%)$. Because of the small solid angle of extraction, only a small fraction of the photoelectrons emitted into $4 \pi$ sr enter the spectrometer and only electrons with the preset kinetic energy are detected at any time. The velocity-map-imaging (VMI) photoelectron spectrometer overcomes this disadvantage with a $100 \%$ collection efficiency and an excellent energy resolution for electrons with low kinetic energies. ${ }^{6-11}$ However, it has high sensitivity to background electrons. Photoelectrons with a fixed kinetic energy give rise to a two-dimensional extended circle on the VMI screen. Its diameter increases with increasing kinetic energy. This widely distributed signal is difficult to distinguish from the noise arising from background electrons, which is usually uniformly distributed throughout the screen. ${ }^{11}$ For the hemispherical analyzer and the VMI spectrometer, the time structure of the light beam does not play a role. They work with pulsed and continuous light sources, and only detector saturation effects may be of concern in the case of the former.

The third common type of electron spectrometer is time-offlight (ToF) instrument. ${ }^{12-26}$ However, they require a time-zero, 
which is typically given by a pulsed laser, ${ }^{24,26}$ a pulsed discharge lamp, ${ }^{23}$ a synchrotron in single-bunch mode, ${ }^{13,14,17,20,22}$ or a fast shutter. ${ }^{15,16,18,19}$ Similar to VMI, time-of-flight instruments measure the complete spectrum at once. The energy resolution increases with the flight tube length, but due to the smaller solid angle from which electrons are extracted, the detection efficiency decreases. This problem can be overcome by electrostatic refocussing ${ }^{21,22}$ or, much more commonly, by the use of magnetic fields. In a magnetic bottle timeof-flight (MB-ToF) electron spectrometer, a divergent magnetic field guides practically all electrons toward the detector. ${ }^{12-18,20,24-26}$ Similar to VMI, MB-ToF has a collection efficiency close to $100 \%$. Finally, there is another advantage of MB-ToF: the strong magnetic field suppresses background electrons from outside the source volume.

Because of this background suppression, MB-ToF spectrometers are well suited for gas phase experiments with extremely low signal intensities (see, e.g., Refs. 24-26). However, they are inherently pulsed with the ionization event starting the clock for the electron ToF. Typical electron flight times are of the order of several microseconds. Therefore, such instruments are usually found in laboratories with pulsed lasers ${ }^{25,26}$ or at synchrotrons operated in the single bunch mode. . $^{13,14,17,20-22}$ It is possible to combine these spectrometers with continuous or quasicontinuous light sources only if the light beam is chopped by a mechanical device. ${ }^{15,16,18,19}$ Typical frequencies of such devices are of the order of $100 \mathrm{kHz}$. For a quasicontinuous light source such as a synchrotron in the multibunch mode, this means a significant reduction in the duty cycle and the loss of roughly three orders of magnitude of intensity.

Here, we present first results of a modified MB-ToF instrument, which can be operated with a continuous or quasicontinuous photoionization source. It has a theoretical collection efficiency close to $100 \%$ with a similarly high upper limit to the duty cycle. Most importantly for our purposes, it suppresses background electrons quantitatively. The energy resolution increases with the length of the flight tube and $\Delta E / E$ of better than $1 \%$ should be easily attained. The instrument presented here is suitable for standard experiments on neutral gases, for photoelectron photoion coincidence (PEPICO) experiments $^{27}$ using the design proposed by Eland et al., ${ }^{24}$ and for experiments on ion beams such as mass-selected clusters. ${ }^{25,26}$ As will be shown, the relationship between the electron ToF distribution and the (ToF) photoelectron spectrum depends on the operation mode and can be quite complex. In the following, we will refer to the measured ToF traces as ToF distributions, as they are often different from the (ToF) photoelectron spectrum.

\section{THE PHOTOELECTRON TRAP}

The starting point is a conventional MB-ToF as described in numerous earlier experiments with pulsed lasers. ${ }^{25,26,28}$ To combine this instrument with a continuous light source, the electrons inside the MB-ToF are gated by a pulsed electric field. Two metal grids are mounted in the path of the photoelectrons toward the electron detector (Fig. 1) $15 \mathrm{~mm}$ above the ionization volume. The first grid is kept at ground potential so that the center region is always free of electric fields. The second grid $1 \mathrm{~mm}$ behind the first one is set to a sufficiently high negative voltage to reflect the arriving photoelectrons. The reflected photoelectrons travel back and pass again the center region. Now, the strong divergent magnetic field comes into

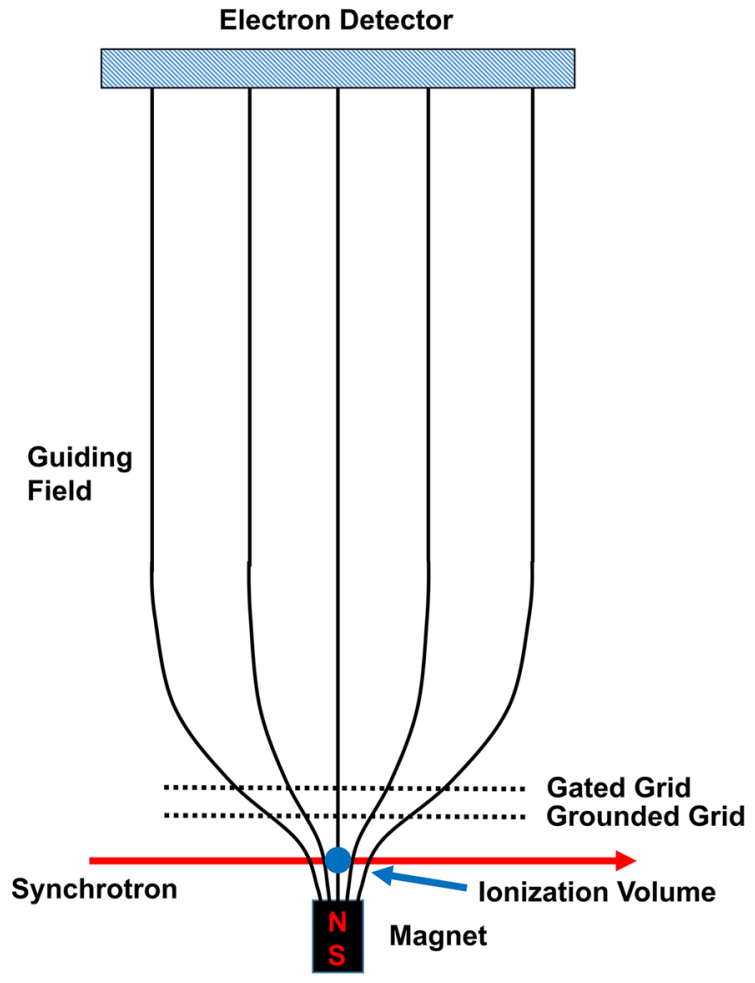

FIG. 1. A conventional magnetic-bottle time-of-flight spectrometer ${ }^{25,26}$ is modified by the insertion of an electrostatic mirror consisting of two grids. If the gated grid is set to a negative voltage the photoelectrons traveling upward are reflected. This electrostatic mirror and the divergent magnetic field together form a trap for the photoelectrons.

play, which increases drastically toward the interaction volume and acts as a magnetic mirror. As a result, photoelectrons are trapped between the magnetic mirror and the electrostatic mirror while they spiral up and down along the magnetic field lines. As time passes, more and more photoelectrons accumulate in this trap. Of course, there are losses due to scattering at the grids and to the nonperfect reflection ability of the magnetic mirror, but the majority of the electrons will survive 5-10 cycles back and forth in the trap. Depending on the lifetime of the electrons inside this trap, the number of accumulated electrons will reach a maximum. Within a few tens of nanoseconds after the electrostatic gate is opened by switching the second grid to ground potential, all electrons will start with their regular path through the drift region of the magnetic bottle and will be detected by the electron detector. The energy resolution is best if the trap is small and the drift region long. After $\approx 0.5 \mu \mathrm{s}$, the trap is closed again and a new cycle of photoelectron accumulation is started. In the following, first results obtained with light from the vacuum ultraviolet (VUV) beamline $e^{29,30}$ at the Swiss Light Source will be presented.

\section{EXPERIMENTAL SETUP}

A new spectrometer has been built at the University of Konstanz and tested at the cluster experiment at the VUV-beamline of 
the Swiss Light Source. ${ }^{11}$ It replaces the VMI spectrometer which has been used previously for the recording of photoelectron spectra of size-selected cluster cations. In a later step, the new spectrometer will be connected to the cluster ion beam apparatus and a cluster ion beam (positive or negative) with a 3-10 nA intensity will be focused into the ionization volume of the spectrometer. The first results presented here have been obtained by introducing neutral gases into the interaction region. The gas pressure has been selected to match the expected target density of the cluster ion beam. In a typical experiment, argon has been used at a pressure of $5 \times 10^{-7} \mathrm{mbar}$. The residual gas pressure was $2 \times 10^{-8}$ mbar. The synchrotron beam of the VUV-beamline is deflected by $75^{\circ}$ using an $\mathrm{Al}$ mirror into the MB-ToF spectrometer and focused to a size of $300 \mu \mathrm{m}$ in the interaction volume of the spectrometer. Depending on the photon energy, the monochromatic flux is $10^{12}-10^{13}$ photons per second. The mirror reduces the intensity by a factor of 20 down to a net flux of $10^{11}$ photons per second.

Aside from the electron trap, the MB-ToF spectrometer is a standard instrument used in previous laser experiments and is described in detail in previous publications. ${ }^{26,28,31}$ The drift region is $1.6 \mathrm{~m}$ long and the guiding field is usually set to $10 \mathrm{G}$. The field strength can be varied over a wide range without observable changes in the photoelectron spectra. The instrument is not shielded with $\mu$-metal. Instead, large Helmholtz coils are used to compensate for external magnetic fields. A conical electric field at the very end of the drift region collects all electrons and guides them to the channeltron. Spectra have been recorded with a standard multistop time-of-light electronics which can be operated at repetition frequencies up to several megahertz. In the electron trap, the strong divergent magnetic field is provided by a commercial cylindrical permanent magnet with $10 \mathrm{~mm}$ diameter and $10 \mathrm{~mm}$ length. The distance to the interaction volume is varied between $1 \mathrm{~mm}$ and $10 \mathrm{~mm}$. The field strength is maximal at the surface of the magnet at $0.5 \mathrm{~T}$ and decreases to $500 \mathrm{G}$ in $10 \mathrm{~mm}$ distance. The grounded grid is mounted $15 \mathrm{~mm}$ and the gated grid $16 \mathrm{~mm}$ above the interaction region.

\section{RESULTS}

\section{A. The three modes of operation}

The modified spectrometer can be operated in three different modes (Fig. 2). If there is no storage effect [Fig. 2(a)], the two grids just act as an electrostatic gate. When the gate voltage is set to zero, electrons can pass through the gate. If all the photoelectrons have the same kinetic energy, the electron detector measures a constant intensity for the time the gate is open, but with a delay corresponding to the time of flight through the drift tube. The spectrum has a rectangular shape with the length equal to the gate opening time. If photoelectrons with a few different kinetic energies are present, the signal intensity increases in steps after opening the gate. Thus, the onset of the ToF distribution corresponds to the integral of the photoelectron spectrum. Therefore, this mode of operation is referred to as the "Integral" mode and the photoelectron spectrum can be obtained by differentiating the rising edge of the ToF distribution. When the gate is closed again, the electron intensity will decrease with a time delay corresponding to the time of flight of the photoelectrons. Differentiation of this region yields the negative photoelectron spectrum.
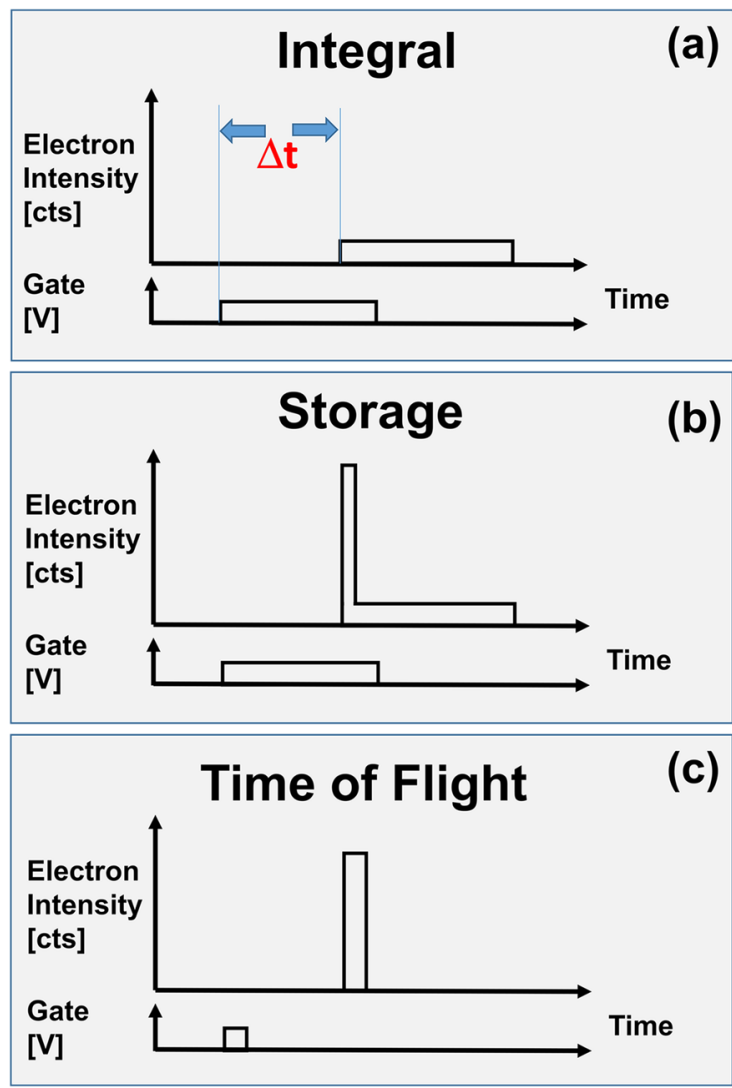

FIG. 2. The three modes of operation of the gated magnetic-bottle time-of-flight electron spectrometer shown together with the ToF distribution of monoenergetic electrons: (a) Integral mode, (b) storage mode, and (c) time-of-flight mode. For an explanation of the different modes, see the text.

When monoenergetic electrons are measured with a storage effect and a long gate opening time [Fig. 2(b)], a sharp peak appears superimposed to the rectangular integral signal. The intensity of the storage peak strongly depends on the details of the design and the accuracy of the fast electric switching of second grid. High frequency oscillations of the grid voltage caused by stray capacitances and inductivities strongly disturb the "storage" peak. It may even vanish. This mode of operation is referred to as the "storage" mode.

In the third mode, the gate is opened for a short time only. The time window must be short compared to the time of flight of the photoelectrons. Since the typical time of flight of photoelectrons with kinetic energies of a few electronvolt is on the order of a few microsecond, gate opening times of a few hundred nanosecond are required in this mode. Now, the recorded ToF distribution resembles the true time-of-flight photoelectron spectrum. However, only a small fraction $\left(\approx 10^{-3}\right)$ of the photoelectrons will be detected without a storage effect. If there is a storage effect, this is the mode of choice. The opening time just needs to be long enough so that all stored electrons are allowed to leave. This time is approximately $0.5 \mu \mathrm{s}$. In this case, the energy resolution does not depend on the opening time, but on the length of the flight path within 
the storage region $(\sim 0.02 \mathrm{~m})$ compared to the length of the drift tube $(1.6 \mathrm{~m})$. The latter can be increased arbitrarily to increase the resolution.

\section{B. Photoelectron spectra: Integral mode}

In the first version of the modified MB-ToF spectrometer, the distance between the interaction volume and the surface of the magnet was only $1 \mathrm{~mm}$, which turned out to be too short for the magnetic mirror to operate. As a result, the storage peak was practically absent from the electron ToF distributions. Figure 3(a) displays an example of a time-of-flight spectrum recorded for argon at $18 \mathrm{eV}$ photon energy and a $5 \mu$ s gate pulse at a repetition rate of $5 \mathrm{kHz}$. The spectrum displayed corresponds to an integral over $10^{5}$ pulses. Time zero is the opening of the gate by switching the voltage from $-10 \mathrm{~V}$ to $0 \mathrm{~V}$. The spectrum is dominated by a rectangular feature with a length of $5 \mu \mathrm{s}$. The delay between zero and the steep onset of the electron signal corresponds to the time-of-flight of electrons with a kinetic energy of $2.3 \mathrm{eV}$, which is the difference between the ionization potential of $\operatorname{Ar}(15.7 \mathrm{eV})^{32}$ and the photon energy of $18 \mathrm{eV}$. As expected, the position of the rectangular

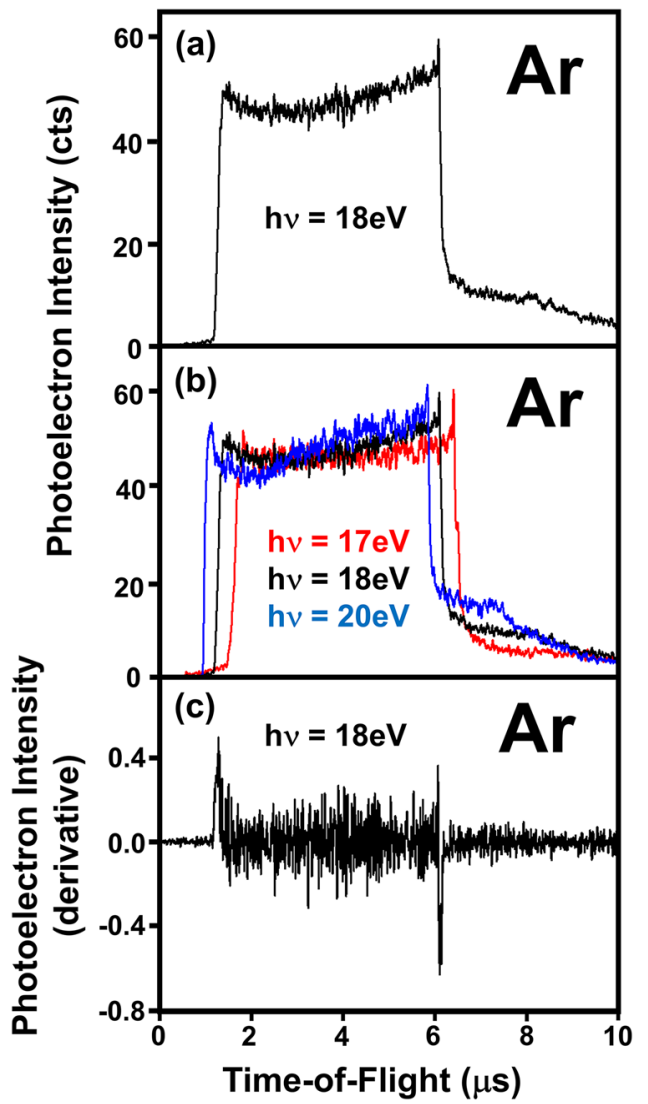

FIG. 3. (a) Photoelectron time-of-flight distribution of argon at a pressure of $5 \times 10^{-7}$ mbar. Time zero is the switching of the gated grid from $-10 \mathrm{~V}$ to $0 \mathrm{~V}$ for $5 \mu \mathrm{s}$. (b) Comparison of the ToF distributions recorded at different photon energies. (c) Photoelectron ToF spectrum obtained by differentiation of the ToF trace shown in (a). feature depends on the photon energy [Fig. 3(b)]. By differentiating the ToF distribution displayed in (a), a narrow peak at a flight time corresponding to a kinetic energy of $2.3 \mathrm{eV}$ appears. This is expected for the integral mode, for which the time-of-flight photoelectron spectrum can be obtained by differentiating the measured trace [Fig. 3(c)].

\section{Photoelectron spectra: Storage mode}

After the first tests, it became clear that the fast pulsing of the gated grid (Fig. 1) is crucial to make the storage peak appear. Hence, the connections between the fast switching electronics outside the vacuum and the grid inside have been improved by using low inductivity and low capacitance cables and feedthroughs. Figure 4(a) displays a spectrum obtained with a $5 \mu$ s gate pulse obtained with the improved electronics. The storage peak at the onset of the rectangular integral spectrum dominates the spectrum. The spectrum displays some additional fine structure, caused by the electronic switching, which will likely disappear when the switching electronics is further improved.

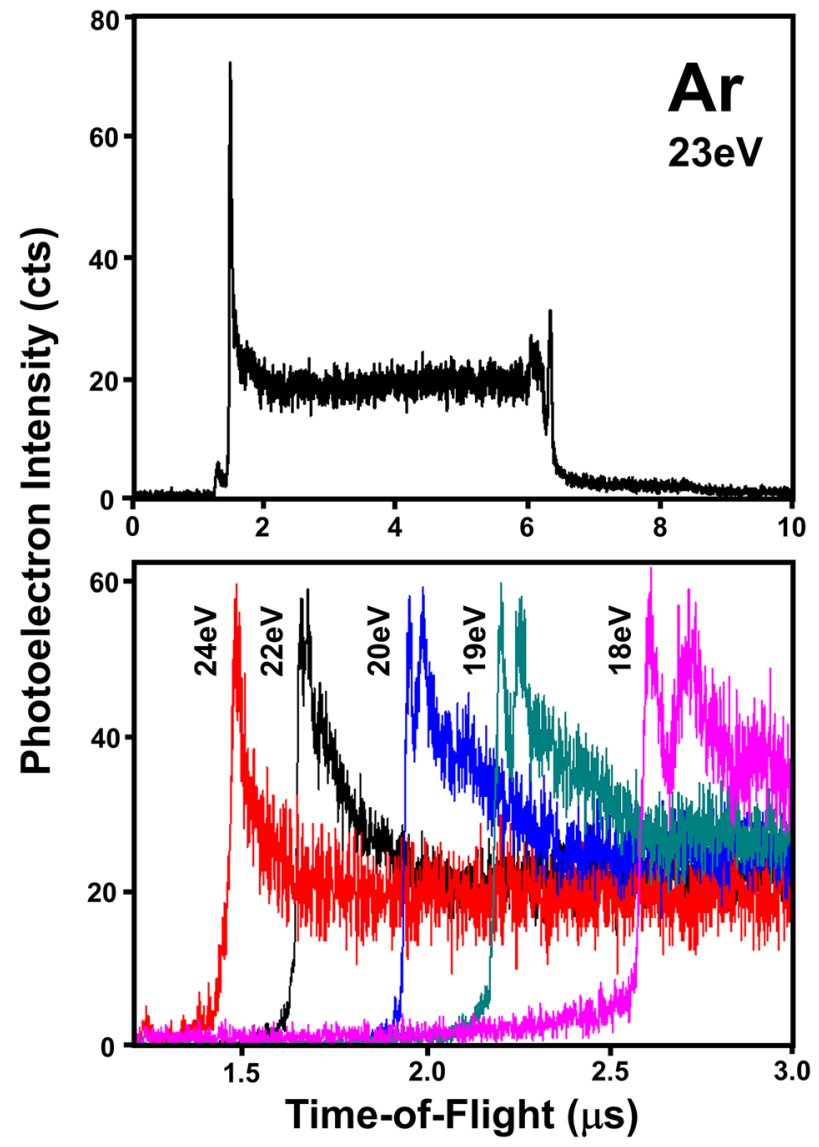

FIG. 4. Top: Photoelectron time-of-flight distribution of Ar obtained with at photon energy of $23 \mathrm{eV}$. Bottom: Dependence of the storage peak on the photon energy. At lower photon energies, the kinetic energy of the electrons decreases and the absolute energy resolution increases. The originally unresolved storage peak splits into the two spin-orbit components of the two final $\mathrm{Ar}^{+}$states, ${ }^{2} P_{3 / 2}$ and ${ }^{2} P_{1 / 2}$. 
The storage peak makes electron time-of-flight spectroscopy practically possible with a (quasi)continuous ionization source. Figure 4(b) displays a series of spectra recorded at different photon energies. At lower kinetic energy of the photoelectrons, the storage peak splits into two components, which are assigned to the two spinorbit components $\left({ }^{2} P_{3 / 2}\right.$ and $\left.{ }^{2} P_{1 / 2}\right)$ of the final state of $\mathrm{Ar}^{+}$. The splitting is known to be $155 \mathrm{meV} .{ }^{33}$ which agrees with the measured ToF difference between the two peaks. The lower energy peak should be smaller by a factor of two. However, the two peaks appear to be about the same since the second one is "sitting" on the asymmetric shoulder of the higher energy peak.

\section{Photoelectron spectra: Time-of-flight mode}

Since the storage peaks contain the photoelectron spectrum, the gate pulse can be shortened to an opening time sufficient to allow the accumulated electron cloud to pass through the two grids. In this mode, the time-of-flight distribution corresponds directly to the electron kinetic energy, only slightly broadened by the remaining integral peak of the shortened gate. Therefore, the ToF distribution should yield the photoelectron time-of-flight spectra directly. Figure 5 shows Ar spectra recorded at photon energies progressively closer to the ionization potential of $15.7 \mathrm{eV}$ using a gate length of $0.5 \mu \mathrm{s}$. The spectra are dominated by two peaks separated by a time of flight corresponding to the $155 \mathrm{meV}$ spin-orbit splitting of the $\mathrm{Ar}^{+}$ground state at an energy resolution of about $40 \mathrm{meV}$. There is an additional feature at $2 \mu$ s flight time (marked X). This is created by closing the gate by applying the gate voltage after the electrons have passed the two grids. The slower electrons, having left the trap shortly before the gate is closed, are accelerated by the field at the back of the gated grid and reach the electron

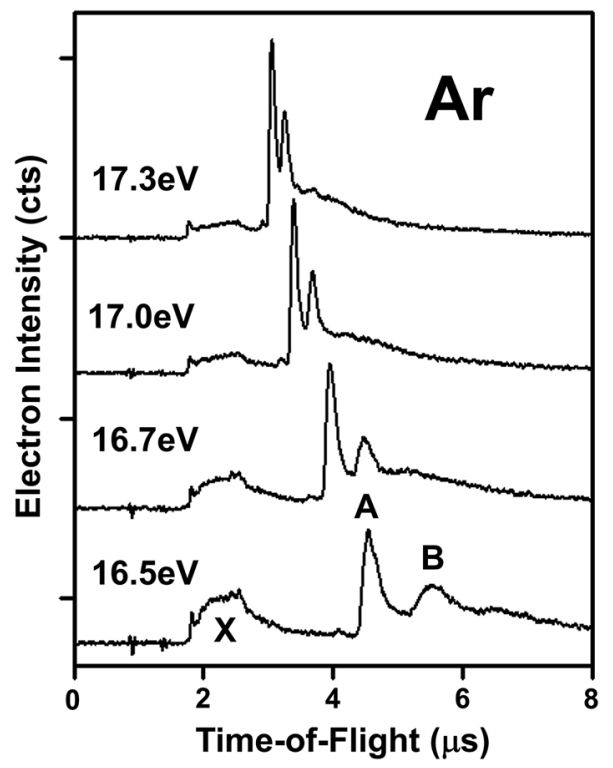

FIG. 5. Argon photoelectron spectra obtained with a gate pulse of $0.5 \mu \mathrm{s}$. The storage peaks dominate the spectra. At photon energies near the ionization potential of $15.7 \mathrm{eV}$, the electrons are slow enough to resolve the spin orbit splitting of $155 \mathrm{meV}$. The feature marked $\mathrm{X}$ is an artifact of the pulsing technique (see the text). detector much earlier. This peak could be suppressed by a third, grounded grid located behind the gated grid. However, we are not convinced such an added complexity is necessary because we understand this artifact and it only interferes with the analysis of very slow electrons.

In principle, an energy resolution of $50 \mathrm{meV}$ should make it possible to resolve vibrational fine structures. The neutral $\mathrm{H}_{2}$ molecule has the largest vibrational quantum of all molecules with an energy separation of $\sim 540 \mathrm{meV} .{ }^{34}$ In photoelectron spectroscopy, the vibrational structure of the final state is observed; in this case, one of the $\mathrm{H}_{2}{ }^{+}$ion. The vibrational progression has a spacing of $\sim 290 \mathrm{meV}$. The new spectrometer was tested for its ability to achieve vibrational resolution by measuring $\mathrm{H}_{2}$. Figure 6 shows two photoelectron spectra obtained in the time-of-flight mode using a gate length of $0.5 \mu \mathrm{s}$. The well-known $\mathrm{H}_{2}{ }^{+}$vibrational structure is reproduced at a photon energy of $17.5 \mathrm{eV}$. The ionization potential of $\mathrm{H}_{2}$ is $15.4 \mathrm{eV} .{ }^{34}$ Hence, the kinetic energy of the electrons of the $0-0$ vibrational transition is $2 \mathrm{eV}$. The spectrum recorded at $16.4 \mathrm{eV}$ is displayed in the lower part of Fig. 6(a). Here, only the first three peaks of the vibrational progression are observed. It should be possible to improve the energy resolution further by improving the gate pulse technique and the drift zone length.

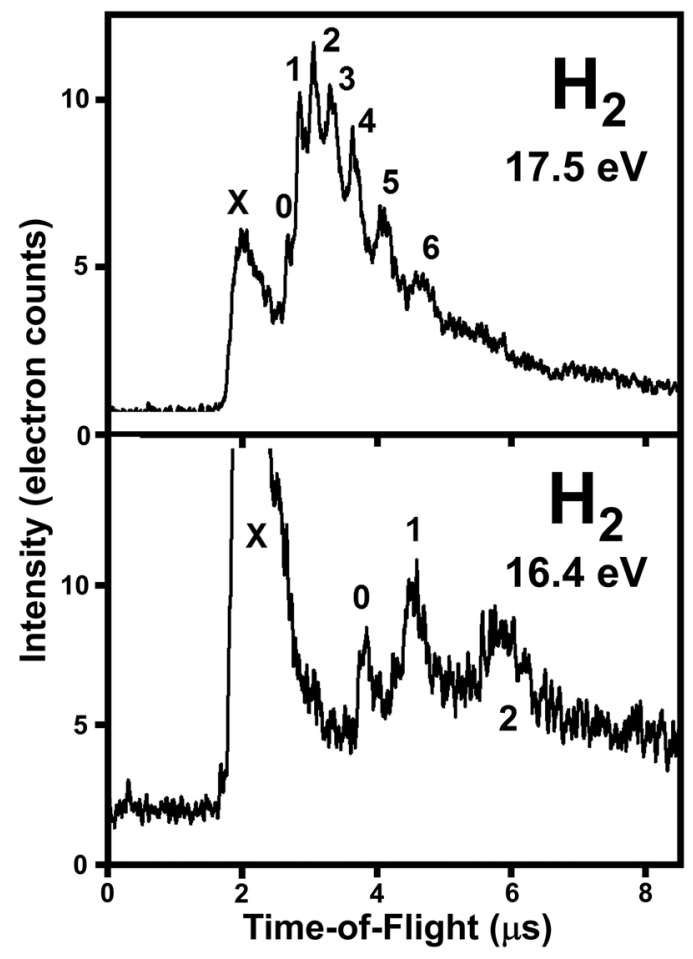

FIG. 6. Upper spectrum: Photoelectron spectrum of $\mathrm{H}_{2}$, obtained with a gate pulse of $0.5 \mu \mathrm{s}$. The vibrational structure with a spacing of $290 \mathrm{meV}$ is clearly resolved (marked 0-6). The feature $X$ is an artifact of the pulse technique and of the same origin as in Fig. 5. Lower Spectrum: At lower photon energy, the electrons slow down and a higher energy resolution is achieved. The peak assigned to the $0-0$ transition shifts from $\sim 2.8 \mu$ s to approximately the position of the $0-4$ transition at $\sim 3.8 \mu \mathrm{s}$, which corresponds to the decrease in photon energy by $1.1 \mathrm{eV}$. 


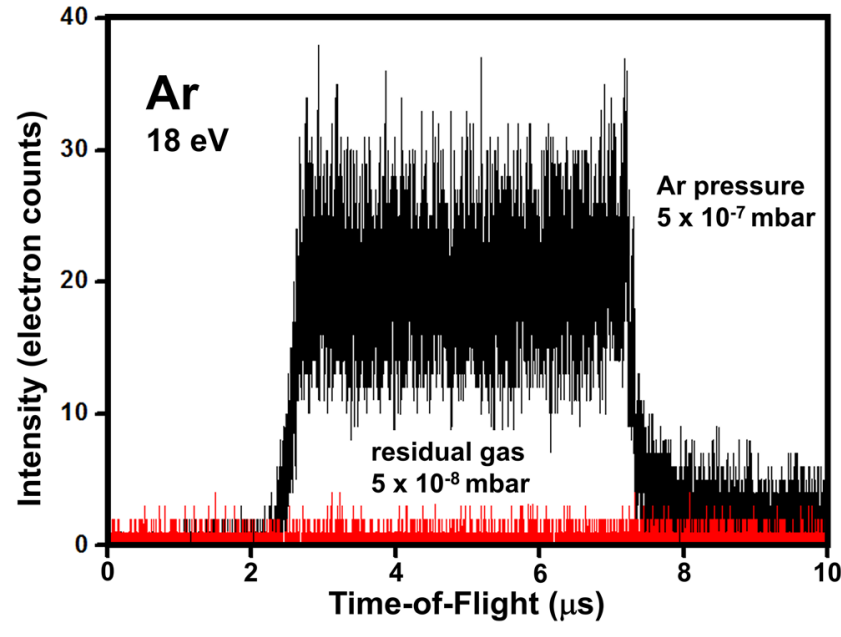

FIG. 7. Comparison of photoelectron ToF traces taken in the integral mode under the same conditions with and without introduction of $\mathrm{Ar}$ gas. The red trace corresponds to background electrons generated either by the ionization of the residual gas or by scattered photons striking surfaces. The background electron signal shows no modulation by the gate pulse.

\section{E. Signal-to-noise ratio}

The synchrotron beam creates background electrons along its path through the spectrometer. There are two main sources: residual gas ionization and secondary electrons from scattered photons hitting surfaces. To measure the signal-to-noise ratio, the residual gas signal at $5 \times 10^{-8}$ mbar is compared with the argon electron ToF distribution recorded at $5 \times 10^{-7} \mathrm{mbar}$ (Fig. 7). The difference in the total number of electrons is a factor of 20 . The electrons recorded without argon show no modulation by the gate lattice.

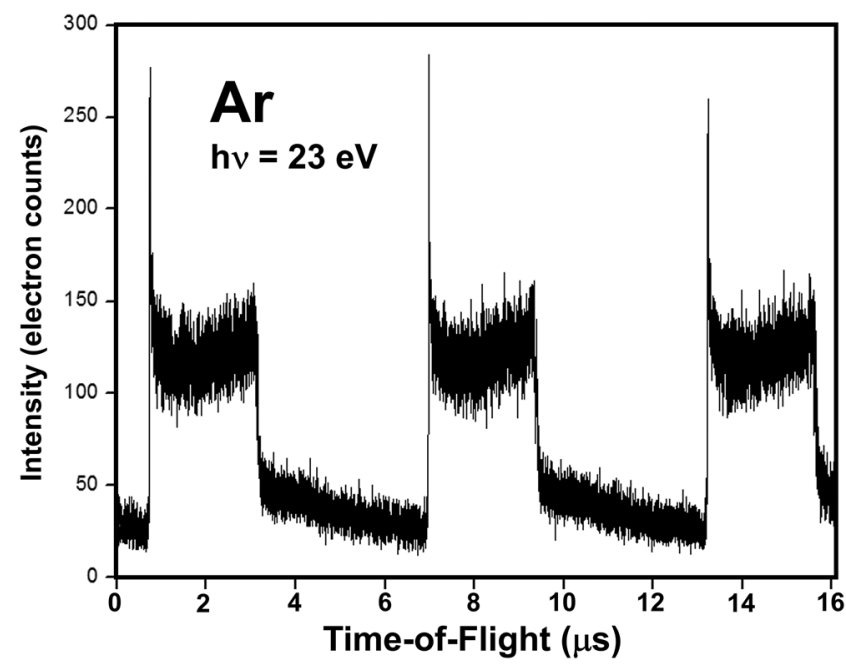

FIG. 8. Time-of-flight photoelectron distributions recorded with a larger time range of $16 \mu \mathrm{s}$ in the storage mode. The gate was operated at $80 \mathrm{kHz}$ so that three pulses appear in the time window. $1 \%$ of all generated photoelectrons are estimated to be detected in the storage peak.
This indicates that these electrons reach the electron detector on irregular paths and not through the gate. Therefore, it may be possible to further improve this signal-to-noise ratio by blocking these paths. But even at this stage, this low signal-to-noise ratio is quite promising.

\section{F. Duty cycle}

In principle, the gate can be pulsed at $100 \mathrm{kHz}$ if a maximum time-of-flight of $10 \mu \mathrm{s}$ is sufficient. So far, the frequency was increased to a maximum value of $80 \mathrm{kHz}$. At this high frequency, the electron count in the storage peak corresponds to $1 \%$ of the total number of electrons in the ToF distribution (Fig. 8). If it is assumed that the magnetic-bottle spectrometer has an efficiency close to $100 \%$ and the magnetic mirror works as expected, this would correspond approximately to $1 \%$ of all generated photoelectrons. At $200 \mathrm{kHz}$, it would be $2.5 \%$. If the storage effect could be further improved by increasing the storage time of the electrons in the trap, an even higher fraction of the photoelectrons could be detected. The limit is yet unknown.

\section{CONCLUSION}

We present first spectra obtained with a modified magnetic bottle time-of-flight electron spectrometer. The new design has three major advantages:

(i) Excellent suppression of background electrons.

(ii) High detection efficiency of all photoelectrons emitted in $4 \pi \mathrm{sr}$.

(iii) Suitable for continuous and quasicontinuous ionization sources.

The key element of the modified spectrometer is an electron trap consisting of the magnetic mirror of the magnetic bottle and a pulsed electrostatic mirror located between the ionization volume and the drift tube of the time-of-flight spectrometer. In the first tests, an energy resolution of $50 \mathrm{meV}$ was achieved with the $1.6 \mathrm{~m}$ long instrument. Although we expected an overall efficiency of more than $10 \%$, only $1 \%$ was achieved at $80 \mathrm{kHz}$. The pulse rate could be further increased up to $\sim 200 \mathrm{kHz}$, resulting in a total electron yield of $2.5 \%$, but this an estimate.

It is not yet clear which effect limits the intensity of the storage peak (Fig. 4, upper trace). One factor may be the transmission of the first grid. In each cycle, electrons pass through this grid twice. At $85 \%$ transmission, this results in a loss of $28 \%$ of all electrons in just one cycle. After 6 cycles, only $20 \%$ survive. The finite transmission of the grids could therefore be the main limitation of the intensity of the storage peak, which was at most four times as large as the continuous signal (upper curve in Fig. 4). To avoid this, a gridless design would be required. However, care will have to be taken that electric field penetration in the ionization region does not reduce the energy resolution and disrupt the electron trajectories. These developments will be part of our future work to explore the potential of this new technique.

\section{ACKNOWLEDGMENTS}

Support by the German Research Foundation is highly appreciated (Project No. GA 389/27-1). The measurements were performed 
at the VUV beamline (Swiss Light Source, Paul Scherrer Institute). A.B. and P.H. acknowledge funding by the Swiss Federal Office of Energy under Contract No. SI/501269-01. The authors are grateful to the infrastructure groups at the SLS for the support and would like to thank Patrick Ascher for his assistance.

\section{REFERENCES}

${ }^{1} \mathrm{~J}$. Berkowitz, Photoabsorption, Photoionization, and Photoelectron Spectroscopy (Academic Press, 2012)

${ }^{2}$ S. Hüfner, "Photoelectron spectroscopy," in Principles and Applications (Springer, Berlin, Heidelberg, 2003).

${ }^{3} \mathrm{H}$. Ibach, "Electron spectroscopy for surface analysis," in Topics in Current Physics (Springer, Berlin, Heidelberg, 1977).

${ }^{4}$ G. Ertl, Low Energy Electrons and Surface Chemistry, Subsequent ed. (VCH Publisher, 1986)

${ }^{5}$ F. Reinert and S. Hüfner, New J. Phys. 7, 97 (2005).

${ }^{6}$ D. W. Chandler and P. L. Houston, J. Chem. Phys. 87, 1445 (1987).

${ }^{7}$ S. J. Cavanagh, S. T. Gibson, M. N. Gale, C. J. Dedman, E. H. Roberts, and B. R. Lewis, Phys. Rev. A 76, 052708 (2007).

${ }^{8}$ G. A. Garcia and L. Nahon, Rev. Sci. Instrum. 76, 053302 (2005).

${ }^{9}$ O. Ghafur, W. Siu, P. Johnsson, M. F. Kling, M. Drescher, and M. J. J. Vrakking, Rev. Sci. Instrum. 80, 033110 (2009).

${ }^{10}$ A. Bodi, P. Hemberger, D. L. Osborn, and B. Sztáray, J. Phys. Chem. Lett. 4, 2948 (2013).

${ }^{11}$ T. Schramm, G. Ganteför, A. Bodi, P. Hemberger, T. Gerber, and B. von Issendorff, Appl. Phys. A 115, 771 (2014).

${ }^{12}$ P. Kruit and F. H. Read, J. Phys. E 16, 313 (1983).

${ }^{13}$ J. H. D. Eland, O. Vieuxmaire, T. Kinugawa, P. Lablanquie, R. I. Hall, and F. Penent, Phys. Rev. Lett. 90, 053003 (2003).

${ }^{14}$ F. Penent, J. Palaudoux, P. Lablanquie, L. Andric, R. Feifel, and J. H. D. Eland Phys, Rev. Lett. 95, 083002 (2005).

${ }^{15}$ J. H. D. Eland, P. Linusson, L. Hedin, E. Andersson, J.-E. Rubensson, and R. Feifel, Phys. Rev. A 78, 063423 (2008).

${ }^{16}$ K. Ito, F. Penent, Y. Hikosaka, E. Shigemasa, I. H. Suzuki, J. H. D. Eland, and P. Lablanquie, Rev. Sci. Instrum. 80, 123101 (2009).
${ }^{17}$ P. Lablanquie, F. Penent, J. Palaudoux, L. Andric, P. Selles, S. Carniato, K. Bucar, M. Zitnik, M. Huttula, J. H. D. Eland, E. Shigemasa, K. Soejima, Y. Hikosaka, I. H. Suzuki, M. Nakano, and K. Ito, Phys. Rev. Lett. 106, 063003 (2011).

${ }^{18}$ J. H. D. Eland, P. Linusson, M. Mucke, and R. Feifel, Chem. Phys. Lett. 548, 90 (2012).

${ }^{19}$ S. Plogmaker, P. Linusson, J. H. D. Eland, N. Baker, E. M. J. Johansson, H. Rensmo, R. Feifel, and H. Siegbahn, Rev. Sci. Instrum. 83, 013115 (2012).

${ }^{20}$ A. Kothe, J. Metje, M. Wilke, A. Moguilevski, R. Al-Obaidi, C. Richter, N. Engel, R. Golnak, I. Y. Kiyan, and E. F. Aziz, Rev. Sci. Instrum. 84, 023106 (2013).

${ }^{21}$ C. Stråhlman, R. Sankari, M. Lundqvist, G. Öhrwall, R. Ovsyannikov, S. Svensson, N. Mårtensson, and R. Nyholm, J. Phys.: Conf. Ser. 425, 092011 (2013).

${ }^{22}$ R. Ovsyannikova, P. Karlsson, M. Lundqvist, C. Lupulescu, W. Eberhardt, A. Fröhlisch, S. Svensson, and N. Martensson, J. Electron Spectrosc. Relat. Phenom. 191, 92 (2013).

${ }^{23}$ C. A. Arrell, J. Ojeda, M. Sabbar, W. A. Okell, T. Witting, T. Siegel, Z. Diveki, S. Hutchinson, L. Gallmann, U. Keller, F. van Mourik, R. T. Chapman, C. Cacho, N. Rodrigues, I. C. E. Turcu, J. W. G. Tisch, E. Springate, J. P. Marangos, and M. Chergui, Rev. Sci. Instrum. 85, 103117 (2014).

${ }^{24}$ A. Hult Roos, J. H. D. Eland, J. Andersson, R. J. Squibb, and R. Feifel, J. Chem. Phys. 149, 204307 (2018).

${ }^{25}$ C.-Y. Cha, G. Ganteför, and W. Eberhardt, Rev. Sci. Instrum, 63, 5661 (1992).

${ }^{26}$ H. Handschuh, G. Ganteför, and W. Eberhardt, Rev. Sci. Instrum. 66, 3838 (1995).

${ }^{27}$ T. Baer and R. P. Tuckett, Phys. Chem. Chem. Phys. 19, 9698-9723 (2017).

${ }^{28}$ A. Koop and G. Gantefor, J. Chem. Phys. 147, 124307 (2017).

${ }^{29}$ M. Johnson, A. Bodi, L. Schulz, and T. Gerber, Nucl. Instrum. Methods Phys. Res., Sect. A 610(2), 597-603 (2009).

${ }^{30}$ A. Bodi, P. Hemberger, T. Gerber and B. Sztáray, Rev. Sci. Instrum. 83, 083105 (2012).

${ }^{31}$ J. Heinzelmann, P. Kruppa, S. Proch, Y. D. Kim, and G. Ganteför, Chem. Phys. Lett. 603, 1 (2014).

${ }^{32}$ See https://physics.nist.gov/PhysRefData/ASD/ionEnergy.html for NIST Atomic Spectra Database Ionization Energies Data. Internet Mar. 25th, 2019.

${ }^{33}$ See https://physics.nist.gov/PhysRefData/Handbook/Tables/argontable6.html for NIST Basic Atomic Spectroscopic data. Internet Mar. 25th, 2019.

${ }^{34}$ L. Asbrink, Chem. Phys. Lett. 7, 549 (1970). 